\title{
Increased Risk of Mortality among Patients Cared for by Physicians with Short Length-of-Stay Tendencies
}

\author{
William N. Southern, MD, MS ${ }^{\top}$ and Julia H. Arnsten, MD, MPH 2,3,4 \\ 'Department of Medicine, Division of Hospital Medicine, Albert Einstein College of Medicine, Montefiore Medical Center, Bronx, NY, USA; \\ 2Department of Medicine, Division of General Internal Medicine, Albert Einstein College of Medicine, Montefiore Medical Center, Bronx, NY, USA; \\ ${ }^{3}$ Department of Psychiatry \& Behavioral Sciences, Albert Einstein College of Medicine, Montefiore Medical Center, Bronx, NY, USA; ${ }^{4}$ Department of \\ Epidemiology \& Population Health, Albert Einstein College of Medicine, Montefiore Medical Center, Bronx, NY, USA.
}

BACKGROUND: Since the introduction of the prospective payment system in 1983, U.S. hospitals have been financially incentivized to reduce inpatient length of stay, and average length of stay has shortened dramatically.

OBJECTIVE: The purpose of this study was to determine whether short length of stay is associated with worse patient outcomes.

DESIGN: We used a quasi-experimental design to compare the outcomes of admissions assigned to physicians with short versus long length-of-stay tendencies. We used each physician's mean length of stay to define their length of stay tendency. We then compared the outcomes of admissions assigned to physicians with short versus long length-of-stay tendencies in propensity score-matched and adjusted analyses using mixed-effects and conditional logistic regression models.

PATIENTS: The study included all admissions for 10 common diagnoses among patients admitted to the medical teaching service of an urban academic hospital from 7/1/2002 through 6/30/2008.

MAIN MEASURE: The primary outcome was 30-day mortality.

RESULTS: We examined 12,341 admissions among 79 physicians. After propensity score matching, admission groups were similar with respect to all demographic and clinical characteristics. Admissions of patients receiving care from short length-of-stay physicians were associated with significantly increased risk of 30-day mortality in adjusted (OR 1.43, 95 \% CI: 1.11-1.85), propensity score-matched (OR 1.33, $95 \% \mathrm{CI}: 1.08-1.63$ ), and matched and adjusted analyses (OR 1.36, 95 \% CI: 0.98-1.90).

CONCLUSIONS: Policies that incentivize short length of stay may lead to worse patient outcomes. The financial benefits of shortening inpatient length of stay should be weighed against the potential harm to patients.

KEY WORDS: Hospital economics; Incentives; Outcomes; Health services. J Gen Intern Med 30(6):712-8

DOI: $10.1007 / \mathrm{s} 11606-014-3155-8$

(c) Society of General Internal Medicine 2015

Received May 13, 2014

Revised September 25, 2014

Accepted December 8, 2014

Published online January 24, 2015

\section{INTRODUCTION}

Since the introduction of the prospective payment system (PPS) in 1983, hospitals in the U.S. have been financially incentivized to shorten inpatient length of stay (LOS). ${ }^{1}$ As facilities have experimented with methods to reduce inpatient LOS, mean LOS for patients hospitalized in the U.S. has fallen dramatically. ${ }^{2,3}$ However, it is unclear whether reducing LOS adversely affects patient outcomes, such as mortality. Given the financial pressures on hospitals to shorten LOS, it is possible that some patients may be discharged too early, and that premature discharge is associated with poorer patient outcomes. To evaluate the PPS, it is essential to examine the effect of short LOS on patient outcomes.

The PPS was introduced by the federal government in 1983 in order to offer financial incentives to hospitals for providing more efficient care. Under the PPS, hospitals are reimbursed a predetermined amount for an episode of inpatient care. Each patient is assigned a diagnosis-related group (DRG) based on clinical information, and the hospital is paid a flat rate for each DRG, regardless of actual services provided. Because hospitals are reimbursed at a fixed rate, lower cost of care (e.g., short LOS) tends to be more profitable, and higher cost of care (e.g., long LOS) less profitable. Partially as a result of the PPS, average LOS for hospitalizations has been reduced dramatically. In 1980, average LOS was 7.3 days, with $30 \%$ of patients hospitalized for 8 days or more. In 2003, average LOS was 4.3 days, with only $16 \%$ of patients staying 8 days or more. Over the same period, the percentage of inpatients staying for 3 days or less increased from $41 \%$ to $57 \%$. Moreover, these reductions in LOS occurred despite a significant increase in the mean age of hospitalized patients. ${ }^{4}$

Although the goal of the PPS is to reduce costs associated with inpatient hospitalizations, it is unclear whether shorter LOS has the unintended consequence of poorer patient outcomes. It is possible that some patients are discharged from the hospital too soon, leading to the occurrence of adverse events. The impact of shorter LOS on outcomes is a critical factor in evaluating the PPS, and more specifically with regard to policies that incentivize short LOS. $^{5-7}$ If such policies are associated with worse patient outcomes, the potential 
associated cost savings must be weighed against the possible harm to patients.

In one study, short LOS was associated with an increased risk of readmission, ${ }^{8}$ and in another it was linked to an increased risk of mortality in patients admitted with acute pulmonary embolism. ${ }^{6}$ Other studies, however, failed to find harm associated with short LOS in patients admitted for acute myocardial infarction $^{9}$ or coronary artery bypass graft surgery $(\mathrm{CABG}),{ }^{10}$ and in Medicare recipients. ${ }^{5}$ Thus, although some authors have suggested that there is a "moral minimum" for LOS, ${ }^{11}$ there is little convincing evidence that short LOS worsens patient outcomes.

Previous attempts to examine associations between LOS and patient outcomes have generally been confounded by patient-level factors. ${ }^{5,6,8-10}$ In particular, because LOS is closely associated with disease severity, patients with shorter LOS are, on average, less severely ill than patients with longer hospitalizations. Observational studies comparing outcomes between the two groups are thus biased toward finding better outcomes for short LOS patients. As such, observational research may fail to reveal the true harm of reducing LOS, largely as a result of patient-level confounding caused by selection bias.

To address the threat of confounding, we used a unique study design that focused on physician LOS tendencies as a grouped treatment variable. ${ }^{12}$ Our objective was to examine outcomes of inpatients assigned to short versus long LOS physicians. We hypothesized that patients under the care of short LOS physicians were at increased risk of 30-day mortality.

\section{METHODS}

\section{Study Setting}

Weiler Hospital is a 381-bed facility that is one of three hospitals comprising Montefiore Medical Center, the primary teaching site of the Albert Einstein College of Medicine of Yeshiva University in New York City.

\section{Study Design}

We used a quasi-experimental design to take advantage of a naturally occurring experiment at Weiler Hospital. In this setting, patients are assigned to one of six identical teams at the time of admission by the admitting resident. In our study, patients assigned to nonteaching teams were excluded. Although teaching team assignment appears random, because allocation is not concealed, we considered this a quasi-experimental design. ${ }^{13,14}$ Eighteen hospitalist and 61 non-hospitalist faculty serve as ward teaching attending physicians, and are assigned to teaching teams in one-month blocks. Because we hypothesized that premature discharge might cause harm among patients under the care of short LOS physicians, we attributed each admission to the discharging attending physician, whom we assumed was the final arbiter of the decision to discharge.

To address the threat of confounding wherein patients assigned to short LOS physicians might be less sick than those assigned to long LOS physicians, we performed both mixed-effects logistic regression modeling, adjusting for patient- and physician-level covariates associated with the two outcomes, and propensity score matching of admission groups.

\section{Study Population}

We examined admissions records for patients with 10 common conditions who were admitted to Weiler Hospital's general medical teaching service from 7/1/2002 through 6/30/2008. The 10 conditions were the most common reasons for hospitalization at the Weiler Hospital medical service during that period of time (congestive heart failure, acute myocardial infarction, chest pain/other coronary, pneumonia, asthma/COPD, diabetes, arrhythmia, sepsis, gastrointestinal bleeding, and HIV). Because we suspected that attending physicians might behave differently with their established patients in ways that could confound our analysis, we excluded inpatients who had had prior contact with the attending physician to whose team they were assigned. In addition, because many patients had more than one admission during the study period, we use the term admission rather than patient to describe our unit of analysis. In order to use all available data, we chose to include all admissions for each patient and to model the clustering of multiple admissions per patient rather than to randomly select a single admission for each patient.

\section{Outcome Measures}

The primary outcome, 30-day mortality, was calculated from the date of admission. We also examined each admission's disposition at discharge, which was classified in one of three categories: home (self-care), home care, or skilled nursing facility. Mortality and other admission-level data (described below) were extracted from Montefiore's clinical information system (CIS) using the Clinical Looking Glass ${ }^{\circledR}$ tool. ${ }^{15}$ These data were merged with the Social Security Death Index, which allowed extraction of mortality after discharge. 


\section{Major Independent Variable}

Given the likelihood that short LOS patients are less sick than long LOS patients, we were unable to examine unbiased associations between LOS and outcomes by means of traditional observational studies. To eliminate bias, we introduced a grouped treatment variable, physician LOS tendency. Our major independent variable, therefore, was the LOS tendency of the attending physician to whom the admission was assigned.

First, in order to moderate the disproportionate effect of a single LOS outlier in terms of a physician's LOS tendency, LOS was truncated to the mean plus 3 standard deviation (22 days). Next, we calculated mean LOS for each physician during the study period in order to define each physicians's LOS tendency. Each physician was designated as either a short LOS physician or a long LOS physician, with the division defined by the median LOS for all physicians. We then created two admission groups (long LOS and short LOS), defined according to the physician to whom the admission had been assigned.

\section{Admission-Level Covariates}

We examined the following patient-level covariates for each admission: age, sex, race/ethnicity, insurance (categorized as Medicare, Medicaid, commercial, or self-pay), and primary discharge diagnosis from the current admission.

We also included two common comorbidity indices: the Charlson comorbidity score ${ }^{16,17}$ and the number of Elixhauser comorbidities. ${ }^{18}$ Albumin and creatinine values were also used as surrogates for overall illness severity. ${ }^{13,19-23}$ In addition, we calculated the Laboratory-based Acute Physiology Score (LAPS) to measure the severity of illness. ${ }^{24,25}$

To create diagnostic variables, each admission's primary ICD-9 code was classified using the Healthcare Cost and Utilization Project database. ${ }^{26}$ For this analysis, we included patients admitted with any of the 10 most frequent reasons for hospitalization at Weiler hospital (congestive heart failure, myocardial infarction, chest pain/other coronary, pneumonia, asthma/COPD, diabetes, arrhythmia, sepsis, gastrointestinal bleeding, and HIV). To adjust for potential differences in mix of diagnoses between the long and short LOS patient populations, we included these diagnostic variables in our adjustment and propensity score models.

To address the threat that a temporal trend might confound our analysis, we included study-year variables in our adjustment and propensity score models.

\section{Physician-Level Covariates}

To determine any similarities between short LOS and long LOS physicians, and to adjust for physician characteristics, we examined several covariates for each physician. Physician inpatient experience was measured by calculating the total number of admissions for each physician over the study period. Other physician-level covariates included hospitalist/nonhospitalist status, years of licensure, and number of years since graduation. We also classified each physician's medical school as U.S. or international. Date of New York State licensure, medical school, and year of medical school graduation were obtained from the New York State Physician Profile website. ${ }^{27}$

\section{Propensity Score-Matched Admission Groups}

Because of differences in admissions assigned to short versus long LOS physicians with respect to certain covariates, we performed propensity score-matched analysis to further reduce the threat of confounding. First, using all admissions, we calculated a propensity score for the likelihood of being assigned to a short LOS physician for each admission, using a non-parsimonious logistic regression model. ${ }^{28,29}$ The model included demographic characteristics, lab values, measures of comorbidity and severity, discharge diagnosis, and year of admission.

One propensity score-matched short LOS admission was selected for each admission assigned to a long LOS physician, using a nearest neighbor greedy matching protocol without replacement. ${ }^{30}$ To minimize matching bias, data were randomly sorted prior to matching. The matching protocol was repeated in sensitivity analyses, and associations between physician LOS tendency and mortality or readmission were estimated after different random sorts.

Of the 12,341 admissions examined, 1,066 (8.6\%) were missing an albumin value, and $35(0.3 \%)$ were missing a creatinine value. The missing values were imputed at the median for the study population (3.8 for albumin and 1.1 for creatinine). Sensitivity analysis excluding discharges with imputed values did not change the estimated association between physician LOS tendency and mortality or readmission.

\section{Statistical Analysis}

Admissions assigned to short and long LOS physicians were first compared with respect to demographic characteristics, type of insurance, laboratory values, comorbidity measures, LAPS score, and discharge diagnosis, using t tests, MannWhitney, or chi-squared tests, as appropriate. The proportion of discharges to home (self-care), home care, and skilled nursing facilities for admissions assigned to short versus long LOS physicians was compared using chi-squared tests.

To assess the association between physician LOS tendency and the dichotomous outcomes of 30-day mortality, we compared rates between the two admission groups in univariate analyses using chi-squared tests. 
Multivariate Analysis of Mortality. \To assess the independent association between physician LOS tendency and mortality using the actual unmatched admission groups, we constructed mixed-effects logistic regression models, including a physicianlevel random effect. We constructed both unadjusted and covariate adjusted models. In these models, we adjusted for both the patient-level and physician-level covariates described above and for the propensity score. Variables were excluded from the models if they were collinear with other independent variables.

Propensity Score-Matched Multivariate Analysis of Readmission and Mortality. We used propensity scored-matched admission groups as an alternative method for examining the independent association between LOS tendency and outcomes, using conditional logistic regression models that included a grouping variable identifying the matched pairs. We did not adjust for patient-level covariates, as these were balanced between the two propensity score-matched admission groups. Because physician-level covariates were not included in the propensity score model and were not balanced in the matched admission groups, we constructed an additional set of conditional logistic regression models adjusting for physicianlevel characteristics in order to address the threat of physician- level confounding. We excluded variables that were collinear with other independent variables.

Stata/IC software version 10.0, (StataCorp, College Station, TX, USA) was used for all statistical analysis and data management. The study was approved by the Montefiore institutional review board.

\section{RESULTS}

\section{Study Population}

Of 14,585 admissions to the Weiler teaching service, 2,244 (15.4\%) had had previous contact with their admitting physician and were excluded, leaving 12,341 admissions and 79 physicians in the study population. The 40 physicians with the shortest LOS were designated short LOS physicians (mean LOS: 3.714.76 days), and the 39 physicians with the longest LOS were designated long LOS physicians (mean LOS: 4.78-8.14 days). A total of 8,759 admissions were assigned to short LOS physicians and 3,582 to long LOS physicians. Patients assigned to short LOS physicians were more likely to be black and male, and had a higher mean Charlson score, more Elixhauser diagnoses, lower mean albumin level, and lower mean LAPS (Table 1).

Table 1. Characteristics of admissions to Long LOS and Short LOS physicians

\begin{tabular}{|c|c|c|c|c|}
\hline & All patients & Long LOS & Short LOS & \\
\hline LOS range (days) & $3.71-8.14$ & $4.78-8.14$ & $3.71-4.76$ & \\
\hline Number of admissions & $(n=12,341)$ & $(n=3,582)$ & $(n=8,759)$ & \\
\hline Number of physicians & $(n=79)$ & $(n=39)$ & $(n=40)$ & $p$ value \\
\hline \multicolumn{5}{|l|}{ Characteristic } \\
\hline Age & $63.4(17.1)$ & $63.1(17.2)$ & $63.5(17.0)$ & 0.21 \\
\hline Male & $5270(42.7)$ & $1477(41.2)$ & $3793(43.3)$ & 0.03 \\
\hline \multicolumn{5}{|l|}{ Race } \\
\hline White & $2710(22.0)$ & $814(22.7)$ & $1896(21.6)$ & 0.19 \\
\hline Black & $3749(30.4)$ & $1006(28.1)$ & $2743(31.3)$ & $<0.001$ \\
\hline Hispanic & $4907(39.8)$ & $1449(40.4)$ & $3458(39.5)$ & 0.32 \\
\hline Other/Unknown & $975(7.9)$ & $313(8.7)$ & $662(7.6)$ & 0.03 \\
\hline \multicolumn{5}{|l|}{ Insurance } \\
\hline Medicare & $4742(38.5)$ & 1317 (36.9) & $3425(39.2)$ & 0.02 \\
\hline Medicaid & $5022(40.8)$ & $1465(41.0)$ & $3557(40.7)$ & 0.74 \\
\hline Commercial & $2374(19.3)$ & $728(20.4)$ & $1646(18.8)$ & 0.05 \\
\hline Self & $168(1.4)$ & $60(1.7)$ & $108(1.2)$ & 0.05 \\
\hline Charlson score & $2.20(2.02)$ & $2.12(2.00)$ & $2.24(2.03)$ & $<0.001$ \\
\hline \# Elixhauser diagnoses & $2.79(1.65)$ & $2.67(1.61)$ & $2.85(1.67)$ & $<0.001$ \\
\hline Admission albumin* & $3.74(0.60)$ & $3.77(0.61)$ & $3.73(0.59)$ & $<0.001$ \\
\hline Admission creatinine $\uparrow$ & $1.1(0.9-1.5)$ & $1.1(0.9-1.5)$ & $1.1(0.8-1.5)$ & 0.005 \\
\hline LAPS $\div$ score & $25.2(19.3)$ & $26.3(19.8)$ & $24.7(19.1)$ & $<0.001$ \\
\hline \multicolumn{5}{|l|}{ Diagnoses, $\S$ No. $(\%)$} \\
\hline $\mathrm{CHF}$ & $2280(18.5)$ & $659(18.4)$ & $1621(18.5)$ & 0.89 \\
\hline Myocardial infarction & $958(7.7)$ & $292(8.1)$ & $666(7.6)$ & 0.30 \\
\hline Chest pain/other coronary disease & $2850(23.1)$ & $886(24.7)$ & $1964(22.4)$ & 0.006 \\
\hline Pneumonia & $1196(9.7)$ & $365(10.2)$ & $831(9.5)$ & 0.23 \\
\hline Asthma/COPD & $1604(13.0)$ & $426(11.9)$ & $1178(13.4)$ & 0.02 \\
\hline Diabetes & $973(7.9)$ & $263(7.3)$ & $710(8.1)$ & 0.15 \\
\hline Arrythmia & $831(6.7)$ & $238(6.6)$ & $593(6.8)$ & 0.80 \\
\hline Sepsis & $784(6.4)$ & $203(5.7)$ & $581(6.6)$ & 0.05 \\
\hline Gastrointestinal bleeding & $398(3.2)$ & $121(3.4)$ & $277(3.2)$ & 0.54 \\
\hline HIV & $467(3.8)$ & $129(3.6)$ & $338(3.9)$ & 0.50 \\
\hline
\end{tabular}

Continuous variables reported as mean (standard deviation) and compared using t tests with unequal variances Dichotomous variables reported as number (percent) and compared using chi-squared tests Creatinine reported as median (interquartile range) and compared using Wilcoxon rank-sum *9.2\% of discharges did not have an albumin value available and were imputed as normal †0.3\% of discharges did not have a creatinine value available and were imputed as normal tLaboratory-based Acute Physiology Score

$\S$ The 10 most frequent diagnoses among medical inpatients during the study period 
Table 2. Characteristics of Long and Short LOS Physicians

\begin{tabular}{lccr}
\hline \hline LOS Range (days) & $\begin{array}{l}\text { Long LOS } \\
\mathbf{4 . 7 8 - 8 . 1 4} \\
(\boldsymbol{n}=\mathbf{3 9})\end{array}$ & $\begin{array}{l}\text { Short LOS } \\
\mathbf{3 . 7 1 - 4 . 7 6} \\
(\boldsymbol{n}=\mathbf{4 0 )}\end{array}$ & p value \\
& & & \\
\hline Number of admissions & $203(249)$ & $478(461)$ & 0.002 \\
Hospitalist & $3(7.7)$ & $15(37.5)$ & 0.002 \\
Years licensed & $18.9(13.8)$ & $8.1(8.5)$ & $<0.001$ \\
Years since graduation & $22.6(14.5)$ & $11.7(8.8)$ & $<0.001$ \\
International graduation & $6(15.4)$ & $7(17.5)$ & 0.80 \\
\hline
\end{tabular}

Continuous variables reported as mean (standard deviation) and compared using $t$ tests with unequal variances

Dichotomous variables reported as number (percent) and compared using chi-squared tests

Compared to long LOS physicians, short LOS physicians were responsible for more total inpatients, were more likely to be hospitalists, and had fewer years of licensure and years since graduation (Table 2).

Table 3. Characteristics of propensity score-matched admissions to Long LOS and Short LOS physicians

\begin{tabular}{|c|c|c|c|}
\hline & Long LOS & Short LOS & \\
\hline LOS range & $4.78-8.14$ & $3.71-4.76$ & \\
\hline Number of admissions & $(n=3570)$ & $(n=3570)$ & \\
\hline Number of physicians & $(n=39)$ & $(n=40)$ & $p$ value \\
\hline \multicolumn{4}{|l|}{ Characteristic } \\
\hline Age & $63.1(17.2)$ & $63.4(17.0)$ & 0.84 \\
\hline Male & $1473(41.3)$ & $1534(43.0)$ & 0.14 \\
\hline \multicolumn{4}{|l|}{ Race } \\
\hline White & $810(22.7)$ & $799(22.4)$ & 0.75 \\
\hline Black & $1004(28.1)$ & $1012(28.3)$ & 0.83 \\
\hline Hispanic & $1444(40.4)$ & $1453(40.7)$ & 0.83 \\
\hline Other/unknown & $312(8.7)$ & $306(8.6)$ & 0.80 \\
\hline \multicolumn{4}{|l|}{ Insurance } \\
\hline Medicare & $1317(36.9)$ & $1347(37.7)$ & 0.46 \\
\hline Medicaid & $1465(41.0)$ & $1438(40.3)$ & 0.51 \\
\hline Commercial & $728(20.4)$ & $733(20.5)$ & 0.88 \\
\hline Self & $60(1.7)$ & $52(1.5)$ & 0.45 \\
\hline Charlson score & $2.12(1.99)$ & $2.17(2.02)$ & 0.24 \\
\hline \# Elixhauser diagnoses & $2.67(1.61)$ & $2.71(1.62)$ & 0.29 \\
\hline Admission albumin* & $3.77(0.61)$ & $3.77(0.62)$ & 0.32 \\
\hline Admission creatinine $\dagger$ & $1.1(0.9-1.5)$ & $1.1(0.9-1.5)$ & 0.05 \\
\hline \multirow{2}{*}{\multicolumn{4}{|c|}{ Diagnoses,§ No.(\%) }} \\
\hline & & & \\
\hline $\mathrm{CHF}$ & $659(18.5)$ & $648(18.1)$ & 0.74 \\
\hline Myocardial infarction & $291(8.2)$ & $301(8.4)$ & 0.67 \\
\hline Chest pain/other coronary disease & $881(24.7)$ & $871(24.4)$ & 0.78 \\
\hline Pneumonia & $365(10.2)$ & $378(10.6)$ & 0.61 \\
\hline Asthma/COPD & 425 (11.9) & 419 (11.7) & 0.83 \\
\hline Diabetes & $261(7.3)$ & $270(7.6)$ & 0.68 \\
\hline Arrythmia & $238(6.7)$ & $231(6.5)$ & 0.74 \\
\hline Sepsis & $203(5.7)$ & $197(5.5)$ & 0.76 \\
\hline Gastrointestinal bleeding & $119(3.3)$ & $120(3.4)$ & 0.95 \\
\hline HIV & $128(3.6)$ & $135(3.8)$ & 0.66 \\
\hline
\end{tabular}

Continuous variables reported as mean (standard deviation) and compared using t-tests

Dichotomous variables reported as number (percent) and compared using chi-squared tests

Creatinine reported as median (inter-quartile range) and compared using Wilcoxon rank-sum

* $9.2 \%$ of discharges did not have an albumin value available and were imputed as normal

†0.3\% of discharges did not have a creatinine value available and were imputed as normal

t Laboratory-based Acute Physiology Score

$\$$ The ten most frequent diagnoses among medical inpatients during the study period

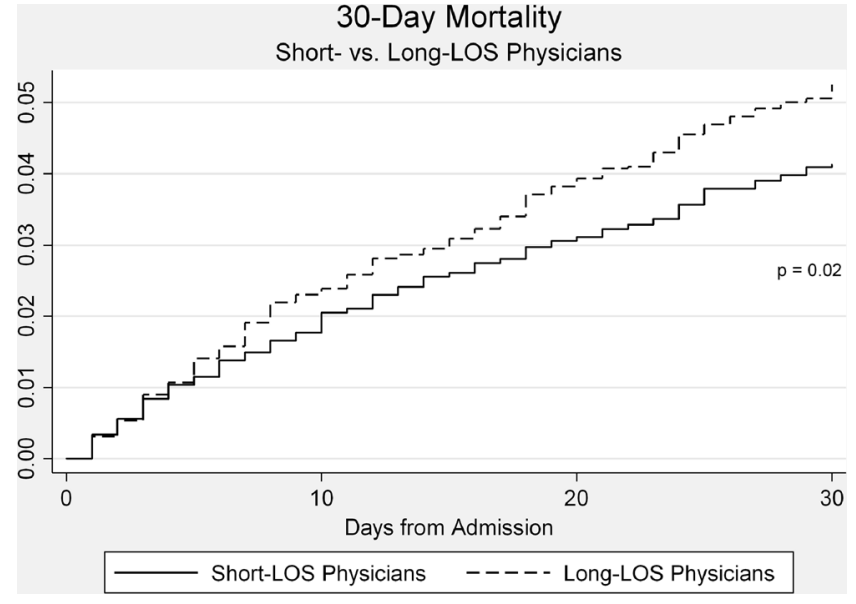

Figure 1. 30-day mortality for patients under the care of short versus long LOS physicians in propensity score-matched cohorts

\section{Propensity Score-Matched Admission Groups}

Of 3,582 admissions assigned to long LOS physicians, propensity scores were calculated for 3,570, and these were matched with 3,570 eligible admissions assigned to short LOS physicians. After propensity score matching, admissions for short and long LOS physicians were similar with respect to age, sex, race/ethnicity, insurance type, admission albumin, admission creatinine, Charlson score, number of Elixhauser diagnoses, LAPS score, and discharge diagnosis (Table 3). When we repeated the matching protocol after different random sorts, the estimated associations between physician LOS tendency and both mortality and readmission were unchanged.

\section{Mortality}

A comparison of the actual unmatched admission groups shows 30-day mortality of $5.5 \%$ (478 deaths) for admissions assigned to short LOS physicians versus $4.3 \%$ (153 deaths) for admissions assigned to long LOS physicians $(p=0.007)$. After

Table 4. Associations between Short LOS Physician Tendency and 30-Day Mortality

\begin{tabular}{ll}
\hline \hline Model & $\begin{array}{l}\text { Mortality } \\
\text { OR (95 \% CI) }\end{array}$ \\
\hline Unadjusted & $1.31(1.07-1.60)$ \\
Propensity score- and covariate-adjusted* & $1.43(1.11-1.85)$ \\
Propensity score-matched $\dagger$ & $1.33(1.08-1.63)$ \\
Propensity score-matched $\dagger$ and covariate-adjusted $\dagger$ & $1.36(0.98-1.90)$ \\
\hline
\end{tabular}

*Adjusted for patient-level characteristics (propensity score, age, sex, racelethnicity, insurance, Charlson comorbidity score, Laboratorybased Acute Physiology Score, and discharge diagnosis) and physician-level characteristics (total number of admissions, hospitalist vs. non-hospitalist, years licensed)

†Propensity score model includes patient-level characteristics (propensity score, age, sex, racelethnicity, insurance, Charlson comorbidity score, Laboratory-based Acute Physiology Score, number of Elixhauser diagnoses, discharge diagnosis, and year of admission)

$\$$ Adjusted for physician-level characteristics (total number of admissions, hospitalist vs. non-hospitalist, years licensed) 


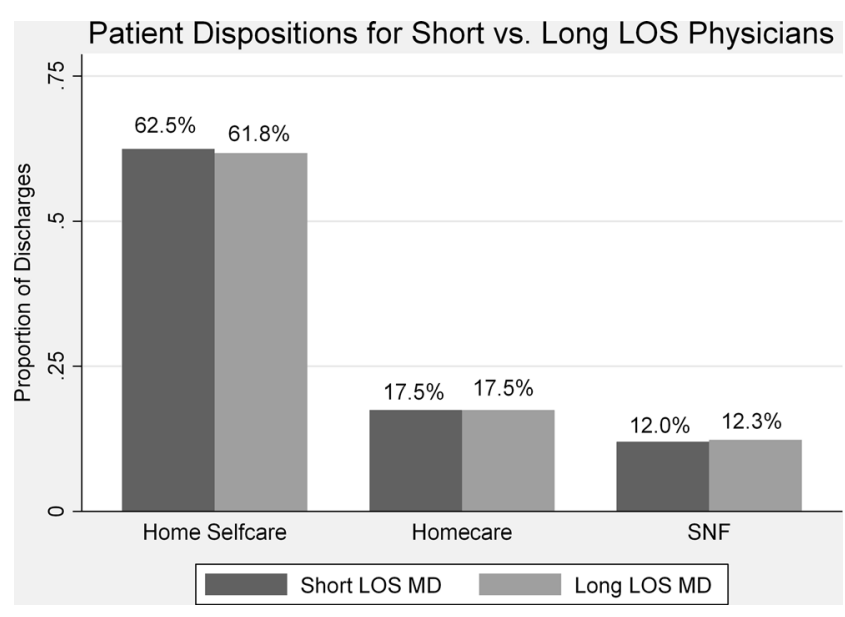

Figure 2. Discharge dispositions for short vs. long LOS physicians

adjusting for propensity score and patient- and physician-level covariates, physician short LOS tendency remained significantly associated with increased risk of 30-day mortality $\left(\mathrm{OR}_{\mathrm{adj}}=1.43,95 \%\right.$ CI 1.11-1.85). In propensity scorematched analyses, physician short LOS tendency was associated with increased risk of 30-day mortality $\left(\mathrm{OR}_{\text {matched }}=1.33\right.$, $95 \%$ CI: 1.08-1.63, Fig. 1), which remained higher after adjusting for physician-level covariates $\left(\mathrm{OR}_{\text {matched/adj }}=1.36\right.$, $95 \%$ CI: 0.98-1.90). These results are summarized in Table 4.

\section{Disposition}

Admissions assigned to short versus long LOS physicians were similarly likely to be discharged home for self-care $(61.8 \%$ vs. $62.5 \%)$, with home care services $(17.5 \%$ vs. $17.5 \%)$, or to skilled nursing facilities $(12.3 \%$ vs. $12.0 \%$, all differences not significant, Figs. 1 and 2).

\section{DISCUSSION}

In a large, carefully controlled retrospective study of 12,341 inpatients at an urban academic medical center, we found that care by a physician with a tendency toward short LOS was associated with a significantly increased risk of death within 30 days. Our findings suggest that a close examination of policies incentivizing short inpatient LOS is warranted. Because policies designed to shorten LOS are aimed at reducing costs associated with inpatient care, the cost savings must be weighed against the potential for worse patient outcomes.

A recent report by Romley et al. found lower rates of inpatient mortality among hospitals that spent more on inpatient care versus hospitals that spent less. ${ }^{31}$ Although evidence suggests that greater overall healthcare spending in the United States does not lead to improved outcomes, ${ }^{32,33}$ Romley et al. suggest that increased spending in one arena, the acute care (inpatient) setting, may lead to an improvement in outcomes among patients. Our findings support the hypothesis that increased intensity and/or duration of inpatient care can result in improved patient outcomes.

Our results may also help to explain some of the conflicting results in studies of hospitalist models of inpatient care. In order to shorten LOS, many hospitals employ hospitalists to care for inpatients in medical ${ }^{13,19,34-36}$ and pediatric ${ }^{37-39}$ services. Some authors have reported a reduction in mortality rates associated with hospitalist care and have suggested that physician inpatient specialization might be beneficial to patients, ${ }^{19,35}$ whereas other research has failed to identify a benefit. ${ }^{13,34,36}$ Our finding that physician tendency toward short LOS, after adjusting for other physician characteristics including hospitalist care, is associated with worse patient outcomes suggests that the potential benefits of hospitalist care (i.e., inpatient specialization) may be offset by the harms associated with shorter LOS.

Despite the strengths of our study, there were some limitations. Because the study was observational in nature, we may not have been able to measure all of the differences between admission groups or between short LOS and long LOS physicians, and there may have been residual patient- or physician-level confounding. To minimize this possibility, we used both logistic regression and propensity score matching to address confounding, and we obtained adjustment variables from a wide range of patient- and physician-level domains. Results were similar between methods, suggesting that residual confounding was minimal. In addition, we were not able to examine readmission as an outcome, as we were unable to assess readmissions to other hospitals. Also, because our outcome measure was linked to the Social Security Death Index, only patients with a Social Security number were included. Finally, this was a single-center study, and the results may not be generalizable.

In conclusion, we found that care by physicians with short LOS tendencies was associated with significantly increased risk of 30-day mortality, suggesting that policies incentivizing short LOS may lead to worse patient outcomes. The cost savings associated with such policies must be weighed against the potential harm to patients that may occur as a result.

Contributors: We would like to thank the Works in Progress Study Group of the Division of General Internal Medicine of Albert Einstein College of Medicine and Montefiore Medical Center for valuable feedback on the analysis and draft of the manuscript.

Financial support: This work was supported by grants UL1 RRO25750, KL2 RRO25749, and TL1 RRO25748 from the Clinical and Translational Science Awards (CTSA) program of the National Center for Research Resources (NCRR), a component of the National Institutes of Health (NIH). Dr. Arnsten was also supported by NIH award number R25 DA023021.

Prior presentations: Portions of this research were presented at the annual meeting of the Society of General Internal Medicine, April 2010, Minneapolis, Minnesota, and at the annual meeting of the Society of Hospital Medicine, April 2010, Washington, DC. 
Conflicts of interest: The authors have no conflicts of interest to report.

Corresponding Author: William N. Southern, MD, MS; Department of Medicine, Division of Hospital MedicineAlbert Einstein College of Medicine, Montefiore Medical Center, 111 East 210th Street, Bronx, NY 10467, USA (e-mail: wsouther@montefiore.org).

\section{REFERENCES}

1. Bunker JP, Schaffarzick RW. Reimbursement incentives for hospital care. Annu Rev Public Health. 1986;7:391-409. 391-409.

2. Kozak LJ, Lees KA, DeFrances CJ. National Hospital Discharge Survey: 2003 annual summary with detailed diagnosis and procedure data. Vital Health Stat. 2006;160:1-206

3. DeFrances CJ, Hall MJ. 2005 National Hospital Discharge Survey. Adv Data. 2007;385:1-19.

4. DeFrances CJ, Lucas CA, Buie VC, Golosinskiy A. 2006 National Hospital Discharge Survey. Natl Health Stat Report. 2008;5:1-20.

5. Baker DW, Einstadter D, Husak SS, Cebul RD. Trends in postdischarge mortality and readmissions: has length of stay declined too far? Arch Intern Med. 2004;164(5):538-544.

6. Aujesky D, Stone RA, Kim S, Crick EJ, Fine MJ. Length of hospital stay and postdischarge mortality in patients with pulmonary embolism: a statewide perspective. Arch Intern Med. 2008;168(7):706-712.

7. Brotman DJ, Lindenauer PK. Length of stay and mortality in pulmonary embolism: high time for evidence-based discharge criteria. Arch Intern Med. 2008;168(7):683-684.

8. Heggestad T. Do hospital length of stay and staffing ratio affect elderly patients' risk of readmission? A nation-wide study of Norwegian hospitals. Health Serv Res. 2002;37(3):647-665.

9. Spencer FA, Lessard D, Gore JM, Yarzebski J, Goldberg RJ. Declining length of hospital stay for acute myocardial infarction and postdischarge outcomes: a community-wide perspective. Arch Intern Med. 2004; 164(7):733-740.

10. Cowper PA, DeLong ER, Hannan EL, et al. Trends in postoperative length of stay after bypass surgery. Am Heart J. 2006;152(6): 1194-1200.

11. Clarke A, Rosen R. Length of stay. How short should hospital care be? Eur J Public Health. 2001;11(2):166-170.

12. Johnston SC, Henneman T, McCulloch CE, van der Laan M. Modeling treatment effects on binary outcomes with groupedtreatment variables and individual covariates. Am J Epidemiol. 2002;156(8):753-760.

13. Southern WN, Berger MA, Bellin EY, Hailpern SM, Arnsten JH. Hospitalist care and length of stay in patients requiring complex discharge planning and close clinical monitoring. Arch Intern Med. 2007; 167(17):1869-1874.

14. Wachter RM, Goldman L. The hospitalist movement 5 years later. JAMA. 2002;287(4):487-494.

15. Clinical Looking Glass. Emerging Health Information Technology; 2009.

16. Deyo RA, Cherkin DC, Ciol MA. Adapting a clinical comorbidity index for use with ICD-9-CM administrative databases. J Clin Epidemiol. 1992;45(6):613-619.

17. Charlson ME, Pompei P, Ales KL, MacKenzie CR. A new method of classifying prognostic comorbidity in longitudinal studies: development and validation. J Chronic Dis. 1987;40(5):373-383.

18. Elixhauser A, Steiner C, Harris DR, Coffey RM. Comorbidity measures for use with administrative data. Med Care. 1998;36(1):8-27.
19. Meltzer D, Manning WG, Morrison J, et al. Effects of physician experience on costs and outcomes on an academic general medicine service: results of a trial of hospitalists. Ann Intern Med. 2002;137(11):866-874.

20. Hollenbeck BK, Miller DC, Taub DA, et al. The effects of adjusting for case mix on mortality and length of stay following radical cystectomy. J Urol. 2006;176(4 Pt 1):1363-1368.

21. Wannamethee SG, Shaper AG, Lennon L, Whincup PH. Height loss in older men: associations with total mortality and incidence of cardiovascular disease. Arch Intern Med. 2006;166(22):2546-2552.

22. Maraldi C, Volpato S, Kritchevsky SB, et al. Impact of inflammation on the relationship among alcohol consumption, mortality, and cardiac events: the health, aging, and body composition study. Arch Intern Med. 2006; 166(14):1490-1497.

23. Goldwasser P, Feldman J. Association of serum albumin and mortality risk. J Clin Epidemiol. 1997;50(6):693-703.

24. Escobar GJ, Greene JD, Scheirer P, Gardner MN, Draper D, Kipnis P. Risk-adjusting hospital inpatient mortality using automated inpatient, outpatient, and laboratory databases. Med Care. 2008;46(3):232-239.

25. van Walraven C, Escobar GJ, Greene JD, Forster AJ. The Kaiser Permanente inpatient risk adjustment methodology was valid in an external patient population. J Clin Epidemiol. 2010;63(7):798-803.

26. Healthcare C, Utilization P, Agency for Healthcare R, Quality RMD. Clinical Classifications Software (CCS). 2007.

27. Health NYSDo. New York State Physician Profile Website. www.nydoctorprofile.com. 2008. www.nydoctorprofile.com.

28. D'Agostino RB Jr. Propensity score methods for bias reduction in the comparison of a treatment to a non-randomized control group. Stat Med. 1998;17(19):2265-2281.

29. D'Agostino RB Jr, D'Agostino RB Sr. Estimating treatment effects using observational data. JAMA. 2007;297(3):314-316.

30. PSMATCH2: Stata module to perform full Mahalanobis and propensity score matching. Boston College School of Economics; 2003.

31. Romley JA, Jena AB, Goldman DP. Hospital spending and inpatient mortality: evidence from California: an observational study. Ann Intern Med. 2011;154(3):160-167.

32. Fisher ES, Wennberg DE, Stukel TA, Gottlieb DJ, Lucas FL, Pinder EL. The implications of regional variations in Medicare spending. Part 2: health outcomes and satisfaction with care. Ann Intern Med. 2003;138(4):288-298.

33. Fisher ES. Medical care-is more always better? N Engl J Med. 2003;349(17):1665-1667.

34. Wachter RM, Katz P, Showstack J, Bindman AB, Goldman L. Reorganizing an academic medical service: impact on cost, quality, patient satisfaction, and education. JAMA. 1998;279(19):1560-1565.

35. Auerbach AD, Wachter RM, Katz P, Showstack J, Baron RB, Goldman L. Implementation of a voluntary hospitalist service at a community teaching hospital: improved clinical efficiency and patient outcomes. Ann Intern Med. 2002;137(11):859-865.

36. Lindenauer PK, Rothberg MB, Pekow PS, Kenwood C, Benjamin EM, Auerbach AD. Outcomes of care by hospitalists, general internists, and family physicians. N Engl J Med. 2007;357(25):2589-2600.

37. Bellet PS, Whitaker RC. Evaluation of a pediatric hospitalist service: impact on length of stay and hospital charges. Pediatrics. 2000;105(3 Pt 1):478-484.

38. Freed GL, Dunham KM, Switalski KE. Assessing the value of pediatric hospitalist programs: the perspective of hospital leaders. Acad Pediatr. 2009;9(3): 192-196.

39. Beck CE, Parkin PC, Friedman JN. Pediatric hospitalist medicine: an overview and a perspective from Toronto, Canada. Clin Pediatr (Phila). 2008;47(6):546-548. 